\title{
Directed, Nickel-Catalyzed Umpolung 1,2-Carboamination of Alkenyl Carbonyl Compounds
}

\author{
Vincent A. van der Puyl, Joseph Derosa, and Keary M. Engle*
}

\begin{abstract}
We report a regioselective, nickel-catalyzed syn-1,2carboamination of non-conjugated alkenyl carbonyl compounds with O-benzoyl hydroxylamine $(\mathrm{N}-\mathrm{O})$ electrophiles and aryl/alkylzinc nucleophiles to afford $\beta$ - and $\gamma$-amino acid derivatives. This method enables preparation of products containing structurally diverse tertiary amine motifs, including heterocycles, and can also be used to form quaternary carbon centers. The reaction takes advantage of a tethered 8-aminoquinoline directing group to control the regiochemical outcome and suppress two-component coupling between the $\mathrm{N}-\mathrm{O}$ electrophile and organozinc nucleophile.
\end{abstract}

Nitrogen-containing small-molecules comprise a significant portion of all known medicines. ${ }^{[1]}$ Thus, novel methods for the formation of carbon-nitrogen $(\mathrm{C}-\mathrm{N})$ bonds have been actively pursued. $^{[2]} 1,2$-Carboamination represents an appealing strategy for converting readily available alkene starting materials into valuable structurally complex amine products in an expedient manner (Scheme 1). This transformation can be carried out using different modes of reactivity, including a classical-polarity approach in which the nitrogen-based reactant functions as a nucleophile (i.e., $\mathrm{R}_{2} \mathrm{NH}$ ) and an umpolung approach where the nitrogen-based reactant is an electrophile (i.e., $\mathrm{R}_{2} \mathrm{NX}, \mathrm{X}=$ halide or pseudohalide), as depicted in Scheme 1a. Catalytic intramolecular (two-component) alkene carboamination involving both polarity types has been extensively studied. ${ }^{[3}$ Intermolecular (three-component) variants, on the other hand, remain comparatively unexplored and have typically been limited to conjugated alkenes (e.g., styrenes or acrylates). ${ }^{[4]}$

In terms of precedents involving non-conjugated, unstrained alkenes, ${ }^{[5]}$ Liu and coworkers have reported palladium-catalyzed carbonylative 1,2-carboamination using 2oxazolidone or phthalimide nucleophiles to afford terminal $\beta$ amino acids. ${ }^{[5]}$ Later, this group reported a similar net transformation involving an azide-containing hypervalent iodine reagent. ${ }^{[5 b]}$ These two reports rely on rapid migratory insertion of $\mathrm{CO}$ to outcompete side reactions, such as $\beta-\mathrm{H}$ elimination. Our group has reported a palladium-catalyzed directed 1,2carboamination of unactivated alkenes via a classical polarity approach (Scheme 1d). ${ }^{[6]}$ In particular, we demonstrated regioselective anti-addition of imides, amides, sulfonamides, and various azaheterocycles with aryl iodides across alkenes. These contributions notwithstanding, 1,2-carboamination of nonconjugated alkenes employing aliphatic amines and alkyl carbon coupling partners remain unexplored. The goal of the present study was to address this knowledge gap through the development of a three-component umpolung carboamination of a non-conjugated alkene using a substrate directivity strategy.

Electrophilic aminating reagents have a rich history in enabling $\mathrm{C}-\mathrm{N}$ bond formation. ${ }^{[7]}$ During the past few years,

[a] V. A. van der Puyl, J. Derosa, K. M. Engle

Department of Chemistry

The Scripps Research Institute

10550 North Torrey Pines Road, La Jolla, California 92037, United

States

E-mail: keary@scripps.edu

Supporting information for this article is given via a link at the end of the document.

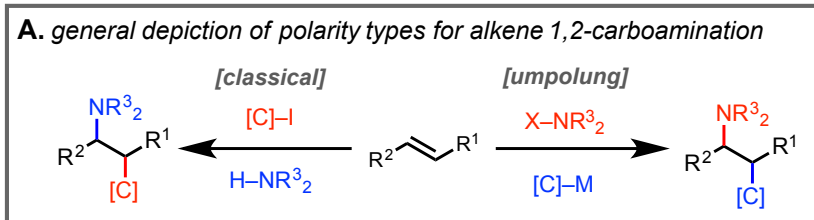

B. intramolecular umpolung 1,2-carboamination precedents

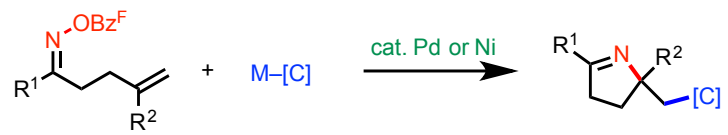

[Bower, 2015]: cat. Pd, B-[C] ([C] = aryl, acyl, vinyl, alkynyl)

[Zhu, 2017]: cat. Pd, H-[C] ([C] = 1,3,4-oxadiazoles)

[Selander, 2017]: cat. Ni, B-[C] ([C] = aryl)

C. intermolecular umpolung radical-based 1,2-carboamination of styrenes

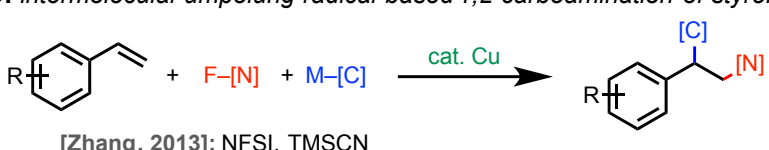

[Zhang, 2013]: NFSI, TMSCN

[Liu, 2017]: F-NMe $\left(\mathrm{SO}_{2} \mathrm{Ph}\right), \mathrm{B}-[\mathrm{C}]([\mathrm{C}]=$ aryl $)$

D. previous work: directed intermolecular classical 1,2-carboamination

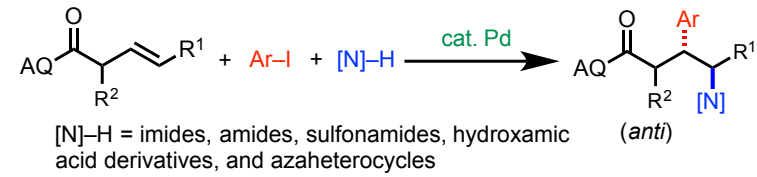

E. this work: directed intermolecular umpolung 1,2-carboamination

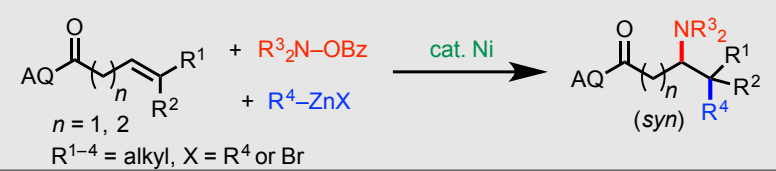

Figure 1. Umpolung Carboamination Background

examples of umpolung carboamination of alkenes and allenes have been described. ${ }^{[4 a, 4 c][8]}$ For example, building on seminal reports by Narasaka, ${ }^{[8]}$ Bower and coworkers described an intramolecular umpolung carboamination of $\gamma, \delta$-unsaturated oxime esters with arylboronic ester coupling partners. ${ }^{[9]}$ The Zhu group later described analogous reactivity with 1,3,4-oxadiazole C-H nucleophiles (Scheme 1b). ${ }^{[10]}$ Regarding intermolecular examples, in 2013 the Zhang group described an umpolung radical-based copper-catalyzed aminocyanation of styrenes employing $\mathrm{N}-\mathrm{F}$ reagents (Scheme 1c). ${ }^{[4 \mathrm{a}]}$ Last year Liu and coworkers published an enantioselective aminoarylation of styrenes also catalyzed by copper using $\mathrm{N}-\mathrm{F}$ reagents as electrophiles. ${ }^{[4 c]}$

In contrast, analogous transformations involving the use of electrophilic aminating reagents in nickel-catalysis have been less extensively studied. ${ }^{[7,}{ }^{11]}$ Two-component nickel-catalyzed $\mathrm{C}-\mathrm{N}$ cross-couplings between organometallic nucleophiles and $\mathrm{N}-\mathrm{O}$ electrophiles have been described by the Johnson, ${ }^{[11 a]}$ Jarvo, ${ }^{[11 \mathrm{~b}]}$ and Knochel groups. ${ }^{[11 \mathrm{c}]}$ To the best of our knowledge, only a single example of nickel-catalyzed alkene carboamination has been reported to date ${ }^{[8 \mathrm{~g}]}$ an intramolecular system developed by Selander and coworkers in 2017 (Scheme 1b). ${ }^{[7 g}$, 11d] Realization of an intermolecular nickel-catalyzed carboamination 
Table 1. Selected Optimization of Reaction Conditions. ${ }^{[a]}$

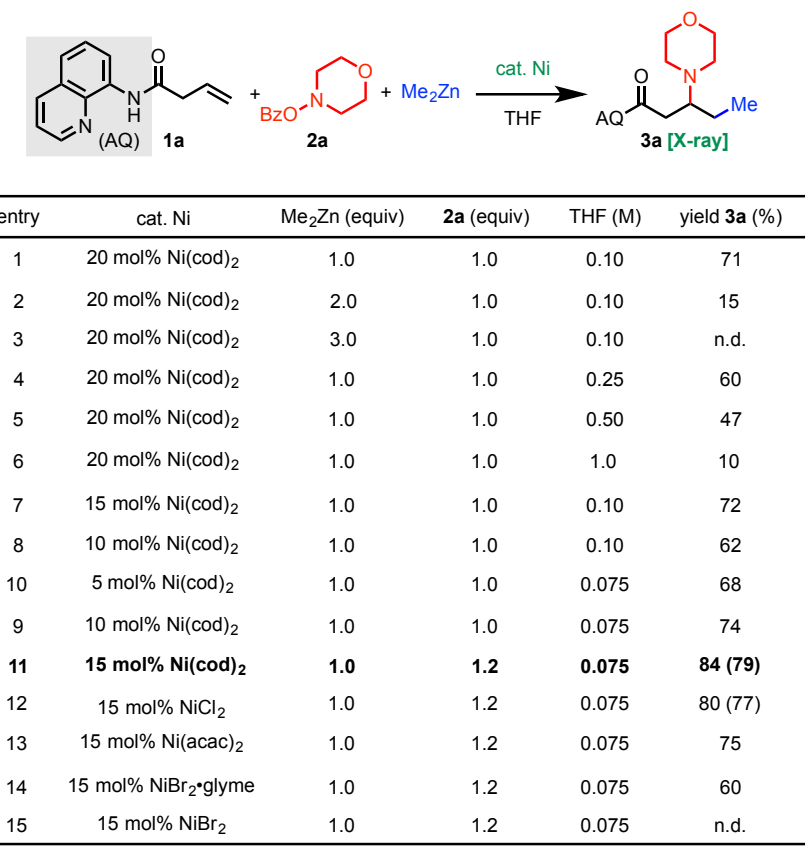

[a] Reaction conditions: 1a $(0.1 \mathrm{mmol}), 2 a\left(1.0-1.2\right.$ equiv), $\mathrm{Me}_{2} \mathrm{Zn}(1.0 \mathrm{M}$ in heptane), $60{ }^{\circ} \mathrm{C}, 18-24 \mathrm{~h} .{ }^{1} \mathrm{H}$ NMR yields reported with $\mathrm{CH}_{2} \mathrm{Br}_{2}$ as internal standard.

process would present the opportunity to rapidly generate medicinally motifs with dense functionality.

We recently described substrate-directed nickel-catalyzed three-component conjunctive cross-coupling reactions ${ }^{[12]}$ that append differentiated alkyl/aryl fragments to $\beta, \gamma-$ and $\gamma, \delta-$ unsaturated carbonyl compounds using aryl/alkyl halides and aryl/alkyl zinc reagents. ${ }^{[13]}$ The regioselectivity of these reactions is controlled by a tethered 8-aminoquinoline (8-AQ) directing group that stabilizes 5 - or 6-membered metallacycles, thereby suppressing undesired side reactions, such as $\beta$-hydride elimination or two-component cross-coupling. Given these results we wondered if it would be possible to employ $O$ benzoylhydroxylamines as electrophiles in lieu of aryl/alkyl halides to synthesize $\beta$ - and $\gamma$-amino acid derivatives under nickel catalysis. We surmised that this approach would complement our previous palladium(II)-catalyzed method (Scheme 1d) in several respects. ${ }^{[6]}$ Namely it would be synselective, proceed with the opposite sense regioselectivity, enable use of alkyl coupling partners, and potentially be compatible with alkenes distal from the $A Q$ group (Scheme 1e).

To test this idea, we elected to use alkene $1 \mathrm{a}$ as the pilot substrate given its unique effectiveness in earlier work ${ }^{[13-14]}$ and $\mathbf{2 a}$ as the electrophilic nitrogen source based on its success in various other catalytic methods. These starting materials were combined with commercially available dimethylzinc solution in the presence of catalytic nickel. With $20 \mathrm{~mol} \% \mathrm{Ni}(\mathrm{cod})_{2}$ we observed formation of product $3 \mathbf{3 a}$ (Table 1) in $71 \%$ yield (Table 1 , Entry 1). DMF, toluene, acetonitrile, and dioxane were also tested under conditions otherwise identical to those in entry 1. The reaction proceeded in toluene and dioxane, though yields were attenuated compared to in THF. Considering conditions from our previous work ${ }^{[13 b]}$ we attempted to drive the reaction to completion by using excess $\mathrm{Me}_{2} \mathrm{Zn}$, but in this case we found significantly diminished yields when more than one equivalent was used. We also found that the reaction was higher yielding at lower concentrations, with the optimal concentration being 0.075 $\mathrm{M}$ 1a in THF. Lower catalyst loadings of $10-15 \mathrm{~mol} \%$ gave comparable yields, though decreasing the catalyst loading further $(5 \mathrm{~mol} \%)$

Table 2. Scope of O-benzoylhydroxylamines and Organozinc reagents. ${ }^{[a]}$

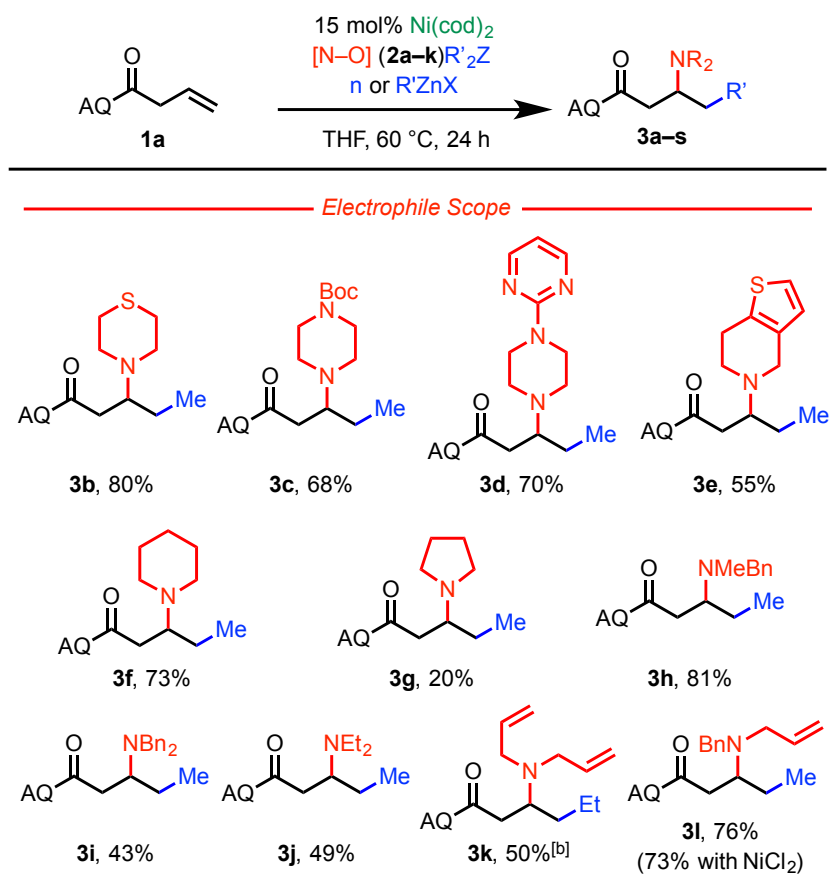<smiles>CCC(CC(=O)OCc1ccccc1)N1CCOCC1</smiles><smiles>O=C(O)CC(CP)N1CCOCC1</smiles><smiles>O=C(O)CC(CC(=O)O)N1CCOCC1</smiles><smiles>CCOC(=O)CC(CBr)N1CCOCC1</smiles>

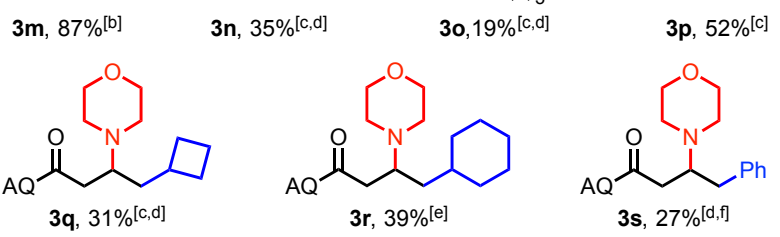

[a] Reaction conditions: 1a (0.2 mmol), 2 (1.2 equiv), $\mathrm{Me}_{2} \mathrm{Zn}$ (1.0 equiv, $1.0 \mathrm{M}$ in heptane), THF $(0.075 \mathrm{M})$. [b] $\mathrm{Et}_{2} \mathrm{Zn}(1.0 \mathrm{M}$ in hexanes). [c] $\mathrm{RZnBr}$ (2 equiv, $0.5 \mathrm{M}$ in THF). [d] $0.1 \mathrm{mmol}$ scale. [e] From dicyclohexylzinc (1.0 equiv, $0.4 \mathrm{M}$ in ether). [f] From solid $\mathrm{Ph}_{2} \mathrm{Zn}$ (1.6 equiv).

led to slightly diminished yield. Increasing the amount of $2 \mathrm{a}$ to 1.2 equiv provided $3 a$ in $84 \%{ }^{1} \mathrm{H}$ NMR yield (79\% isolated). We also found that the reaction performs comparably well using several bench-stable $\mathrm{Ni}(\mathrm{II})$ salts, enabling a glove-box free protocol. The product 3a was isolated in $77 \%$ yield using $\mathrm{NiCl}_{2}$ as the precatalyst.

Having optimized the reaction conditions, we proceeded to explore the O-benzoylhydroxylamine electrophile scope (Table 2). We found that several hetereocyclic motifs (3b-3g) frequently found in bioactive compounds were well-tolerated, including thiomorpholine, tert-butyoxycarbonyl-protected piperazine, 2-(piperazin-1-yl)pyrimidine, 4,5,6,7tetrahydrothieno[3,2-c]pyridine, piperidine, and pyrrolidine. An array of $\mathrm{N}-\mathrm{O}$ reagents derived from acyclic amines $(\mathbf{2 h}-\mathbf{2 l})$, including $\mathrm{N}$-methyl- $\mathrm{N}$-benzylamine, diethylamine, dibenzylamine and diallylamine also reacted under optimized conditions. Sterically hindered and especially reactive $\mathrm{N}-\mathrm{O}$ reagents could not be used as coupling partners in this reaction (see $\mathrm{SI}$ ). The product 3I was obtained in a similar yield using $\mathrm{NiCl}_{2}$, highlighting its efficacy as a substitute for $\mathrm{Ni}(\operatorname{cod})_{2}$. 
The reaction was also compatible with a variety of diorganozinc and organozinc halide nucleophiles, though some reactions were found to proceed in diminished yields. In some cases this could be overcome by slow addition of the organozinc

Table 3. Scope of Alkene Substrates. ${ }^{[a]}$

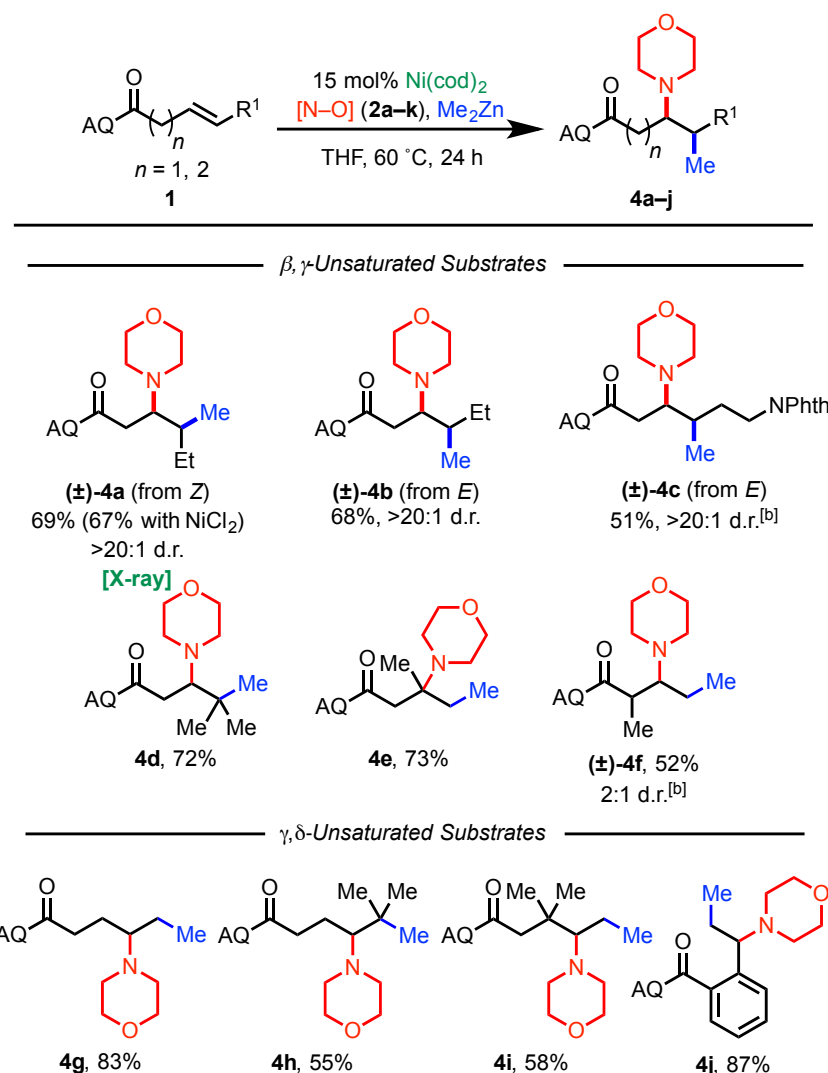

[a] Reaction conditions: 1a (0.2 mmol), 2 (1.2 equiv), $\mathrm{Me}_{2} \mathrm{Zn}$ (1.0 equiv, $1.0 \mathrm{M}$ in heptane), THF $(0.075 \mathrm{M})$. [b] $0.1 \mathrm{mmol}$ scale.

nucleophile, demonstrated in the synthesis of $3 \mathrm{~m}$, which was isolated in $87 \%$ yield. Several other primary alkylzinc nucleophiles were compatible, including propyl (3n), ethyl propionate (3o) and benzyl (3p), providing the corresponding products in moderate yields. Secondary alkylzinc nucleophiles such as cyclobutyl (3q) and cyclohexyl (3r) could also be employed and provided moderate yields. Tertiary carbon nucleophiles were unsuccessful under the optimized reaction conditions (see $\mathrm{SI}$ ). We observed that monoalkylzinc halides were generally lower yielding that dialkylzinc reagents, likely due to their well-known attenuated nucleophilicity. We hypothesize that the need for excess alkylzinc halide (four times more than in the case of dialkylzinc reagents) is due to competitive reduction of the electrophile $\mathbf{2 a}$ before it is able to react in the desired pathway, leading to decreased yields. In the case of secondary nucleophiles, we believe competing $\beta$-hydride elimination pathways generates reducing species in solution that facilitate electrophile decomposition. We have also isolated aminoarylated product 3 s in $27 \%$ yield.

We also explored the scope of alkene substrates (Table 3) and found that the reaction was compatible with a variety of substituted alkenes. The relative stereochemistry of $\mathbf{4 a}$ was determined by X-ray crystallography, establishing that the reaction proceeded in a syn-selective manner. ${ }^{[13]}$ The stereochemistry of other products derived from internal 1,2disubstituted alkenes were assigned by analogy. 4a was also obtained using $\mathrm{NiCl}_{2}$ as the precatalyst and was obtained with similar yield. We also found that a phthalimide-protected amine could be tolerated under the reaction conditions to afford carboaminated product $\mathbf{4 c}$ in moderate yield. Given the success of setting quaternary carbon centers in our previously published dialkylation reaction, ${ }^{[13 \mathrm{~b}]}$ we wondered whether this

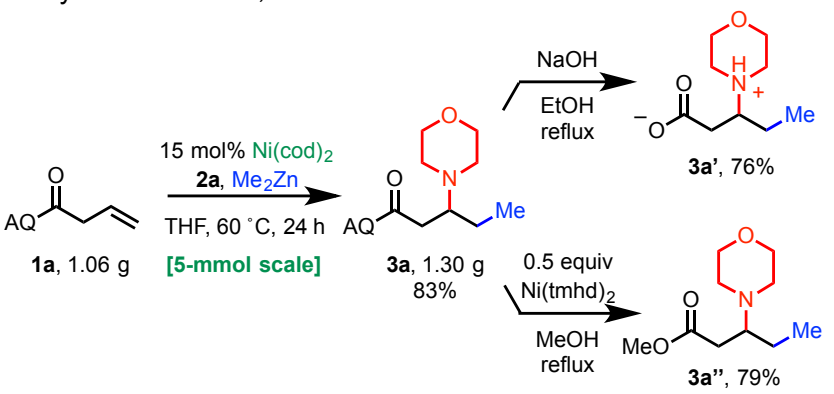

Figure 2. Reaction Scale-Up and 8-Aminoquinoline Removal.

carboamination reaction could also function in sterically congested environments. We were pleased to find tri- and 1,1disubstituted alkenes could be used to synthesize compounds 4d and $4 \mathrm{e}$, respectively, in good yields, demonstrating the ability of this method to forge quaternary carbons centers at either the $\beta$ and $\gamma$ position. The reaction also proceeded in moderate yields with a-methyl substituted alkenyl carbonyl compounds (4f), though we found the benzyl-substituted analogue to proceed in significantly reduced yields ( $<10 \%$ isolated). We also found the reaction could be extended to $\gamma, \delta$-unsaturated substrates to afford products $\mathbf{4 g}-\mathbf{4 i}$ and 2 -vinylbenzamide-derived product $\mathbf{4 j}$.

We next performed the reaction on gram scale to demonstrate its synthetic utility. On $5-\mathrm{mmol}$ scale, we were able to isolate $1.30 \mathrm{~g}$ of $\mathbf{3 a}$ in $83 \%$ yield (Scheme 2). We also validated two methods to remove the $A Q$ directing group.

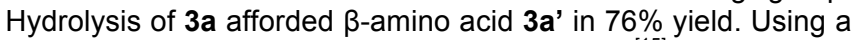
method published by Ohshima and coworkers,${ }^{[15]}$ methanolysis of $\mathbf{3 a}$ afforded ester $\mathbf{3 a}$ " in $\mathbf{7 9 \%}$ yield. Furthermore, we found that a stereocenter at the carbon $\alpha$ to the carbonyl did not racemize under the reaction conditions (see SI for details).

Regarding the reaction mechanism, we surmised that two plausible redox manifolds could be operative, namely $\mathrm{Ni}(0) / \mathrm{Ni}(\mathrm{II})$ or $\mathrm{Ni}(\mathrm{I}) / \mathrm{Ni}(\mathrm{III})$ catalysis (depicted in general form as $\mathrm{Ni}(n) / \mathrm{N}(n+2))$. Moreover, the reaction could proceed via two different orders of events (Fig. 3a). In Pathway A, substratebound nickel complex $6 \mathrm{c}$ would first undergo oxidative addition with 2 to form intermediate $\mathbf{6 a}$. Transmetalation followed by insertion to the alkene would form $\mathbf{6 b}$, which could reductively eliminate to form products $\mathbf{3 - 4}$ and regenerate the active catalyst. In the second potential mechanism, Pathway B, intermediate $6 \mathrm{c}$ would first react via transmetalation, after which migratory insertion would lead to intermediate $\mathbf{6 d}$. This species could then oxidatively add to $\mathbf{2}$ to give nickel intermediate $\mathbf{6 e}$. Reductive elimination would form the key $\mathrm{C}\left(\mathrm{sp}^{3}\right)-\mathrm{N}$ bond and regenerate the catalytically active low-valent nickel species. A third mechanistic scenario (see $\mathrm{SI}$ ) in which $\mathrm{C}-\mathrm{N}$ bond formation precedes transmetalation and $\mathrm{C}-\mathrm{C}$ reductive elimination cannot be conclusively ruled out at this stage, though we consider it to be less likely because it would involve formation of larger nickelacycles in preference to smaller nickelacycles with both classes of substrates (6 versus 5 with products 3 , and 7 versus 6 with products 4 ).

In an effort to disambiguate between these possibilities, we prepared radical clock electrophile $\mathbf{2 m}$ (Fig. 3b). Based on literature precedents, the corresponding aminyl radical-which would be formed if SET oxidative addition were operative ${ }^{[11 d]}$ - 
was expected to cyclize with a first-order rate constant of approximately $10^{4} \mathrm{~s}^{-1} \cdot{ }^{16]}$ When this electrophile was subjected to standard reaction conditions, only non-cyclized product $3 \mathbf{t}$ was formed in $40 \%$ yield. No evidence of cyclization was observed by ${ }^{1} \mathrm{H}$ NMR of the crude reaction mixture. This result is consistent with a two-electron oxidation addition pathway or alternatively with an SET oxidation pathway involving a radical recombination step with a rate constant $>10^{4} \mathrm{~s}^{-1}$.

The effect of radical inhibitors was next studied (Fig. 3c). The reaction was not inhibited by the addition of BHT (1 equiv). On the other hand, addition of TEMPO (1 equiv) dramatically suppressed product formation, leading to unreacted starting materials, as well as TEMPO-H and TEMPO-Me adducts, as monitored by ${ }^{1} \mathrm{H}$ NMR and LC-MS. This result suggests that a $\mathrm{Ni}(\mathrm{I}) /(\mathrm{III})$ cycle involving a $\mathrm{Ni}(\mathrm{I})-\mathrm{Me}$ intermediate and SET events may be operative; however, a more detailed mechanistic study is needed before firm conclusions can be drawn.
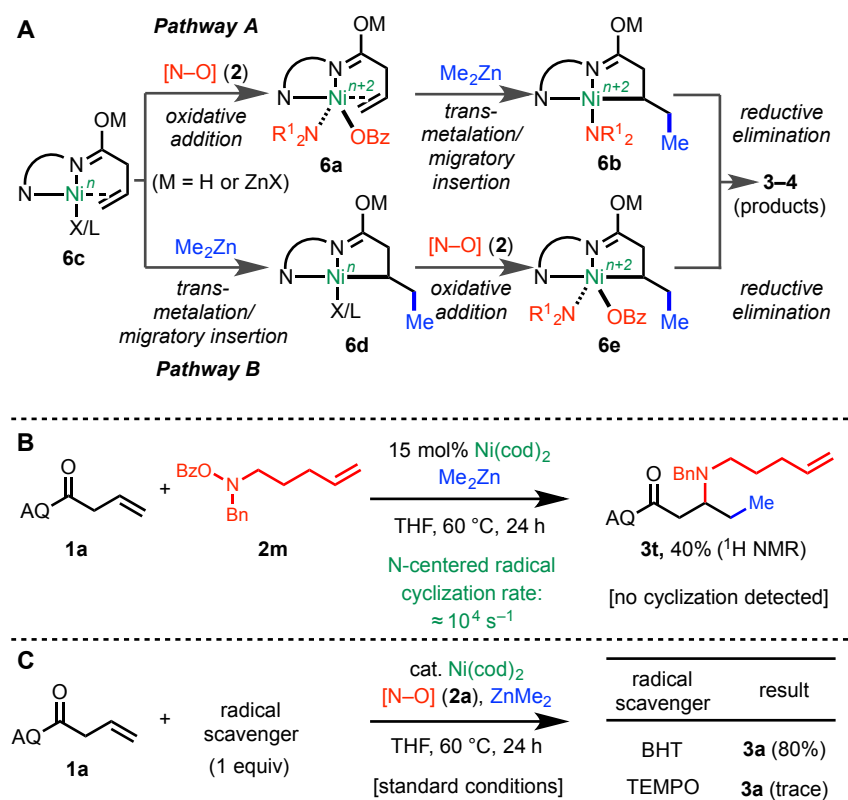

Figure 3. Plausible Pathways and Preliminary Mechanistic Experiments.

In summary, we have developed an intramolecular umpolung carboamination of non-conjugated alkenes that affords a variety of $\beta$ - and $\gamma$-amino acid and ester derivatives. The reaction is enabled by a removable 8-aminoquinoline tethered directing group, which facilitates formation of stabilized 5 - or 6-membered nickelacycles, suppresses $\beta$-hydride elimination and two-component coupling, and determines the regiochemical outcome. The reaction tolerates a range of alkenes with various substitution patterns and proceeds in the presence of several synthetically important functional groups.

\section{Acknowledgements}

This work was financially supported by The Scripps Research Institute (TSRI), Pfizer, Inc., Bristol-Myers Squibb (Unrestricted Grant), and the National Science Foundation (CHE-1800280). We further acknowledge the NSF for a Graduate Research Fellowship (NSF/DGE-1346837, J.D.) and a Summer Undergraduate Research Fellowship (NSF/DBI-1359160,
V.A.V.). We thank Dr. De-Wei Gao, Mingyu Liu, and Van T. Tran for preparation of alkene substrates and nitrogen electrophiles. We also thank Professor Arnold L. Rheingold for assistance with X-ray crystallography.

Keywords: nickel • carboamination • alkene • directing group • electrophilic nitrogen

[1] a) T. Henkel, R. M. Brunne, H. Müller, F. Reichel, Angew. Chem. Int Ed. 1999, 38, 643-647; b) E. Vitaku, D. T. Smith, J. T. Njardarson, J. Med. Chem. 2014, 57, 10257-10274.

[2] a) J. F. Hartwig, Angew. Chem. Int. Ed. 1998, 37, 2046-2067; b) T. E. Müller, M. Beller, Chem. Rev. 1998, 98, 675-703; c) J. P. Wolfe, S. Wagaw, J.-F. Marcoux, S. L. Buchwald, Acc. Chem. Res. 1998, 31, 805-818; d) S. V. Ley, A. W. Thomas, Angew. Chem. Int. Ed. 2003, 42, 5400-5449; e) M. Beller, J. Seayad, A. Tillack, H. Jiao, Angew. Chem. Int. Ed. 2004, 43, 3368-3398; f) R. Wijtmans, M. K. S. Vink, H. E. Schoemaker, F. L. van Delft, R. H. Blaauw, F. P. J. T. Rutjej, Synthesis 2004, 5, 641-662; g) J. F. Hartwig, Nature 2008, 455, 314-322; h) J. Beriwal, E. Van der Eycken, Chem. Soc. Rev. 2013, 42, 9283-9303; i) P. Ruiz-Castillo, S. L. Buchwald, Chem. Rev. 2016, 116, 12564-12649; j) M. Dai, Z. Ye, K. E. Gettys, Synthesis 2017, 49, 2589-2604.

[3] a) S. R. Chemler, Org. Biomol. Chem. 2009, 7, 3009-3019; b) R. I. McDonald, G. Liu, S. S. Stahl, Chem. Rev. 2011, 111, 2981-3019; c) D. M. Schultz, J. P. Wolfe, Synthesis 2012, 44, 351-361; d) Z. J. Garlets, D. R. White, J. P. Wolfe, Asian J. Org. Chem. 2016, 6, 636-653; e) G. M. Belov, D. S. Belov, A. V. Kurkin, M. A. Yurovskya, Chem. Heterocycl. Comp. 2012, 48, 73-81; f) Y. Shimizu, M. Kanai, Tetrahedron Lett. 2014, 55, 3727-3737; g) J. P. Wolfe, Synlett 2008, 19, 2913-2937; h) J. P. Wolfe, Eur. J. Org. Chem. 2007, 571-582.

[4] a) H. Zhang, W. Pu, T. Xiong, Y. Li, X. Zhou, K. Zun, Q. Liu, Q. Zhang, Angew. Chem. Int. Ed. 2013, 52, 2529-2533; b) Y.-Y. Liu, X.-H. Yang, R.-J. Song, S. Luo, J.-H. Li, Nat. Comm. 2017, 8, 14720; c) D. Wang, L. Wu, F. Wang, X. Wan, P. Chen, Z. Lin, G. Liu, J. Am. Chem. Soc. 2017, 139, 6811-6814; d) D. Wang, F. Wang, P. Chen, Z. Lin, G. Liu, Angew. Chem. Int. Ed. 2017, 56, 2054-2058; e) B. Qian, S. Chen, T. Wang, X. Zhang, H. Bao, J. Am. Chem. Soc. 2017, 139, 13076-13082; f) S. N. Gockel, T. L. Buchanan, K. L. Hull, J. Am. Chem. Soc. 2018, 140, 5861.

[5] a) J. Cheng, X. Qi, M. Li, P. Chen, G. Liu, J. Am. Chem. Soc. 2015, 137, 2480-2483; b) M. Li, F. Yu, P. Chen, G. Liu, J. Org. Chem. 2017, 82, 11682-11690.

[6] Z. Liu, Y. Wang, Z. Wang, T. Zeng, P. Liu, K. M. Engle, J. Am. Chem. Soc. 2017, 139, 11261-11270.

[7] a) P. Kovacic, M. K. Lowery, K. W. Field, Chem. Rev. 1970, 70, 639665; b) E. Erdik, M. Ay, Chem. Rev. 1989, 89, 1947-1980; c) E. Ciganek, Electrophilic amination of carbanions, enolates and their surrogates. In Organic Reactions, Hoboken, NJ, United States, 2008; Vol. 72, pp 1-366; d) T. J. Barker, E. R. Jarvo, Synthesis 2011, 24, 3954-3964; e) M. Corpet, C. Gosmini, Synthesis 2014, 46, 2258-2271; f) X. Yan, X. Yang, C. Xi, Catal. Sci. Technol. 2014, 4, 4169-4177; g) X. Dong, Q. Liu, Y. Dong, H. Liu, Chem. Eur. J. 2017, 23, 2481-2511; h) C. E. Hendrick, Q. Wang, J. Org. Chem. 2017, 82, 839-847.

[8] a) A. Faulkner, J. S. Scott, J. F. Bower, J. Am. Chem. Soc. 2015, 137, 7224-7230; b) X. Bao, Q. Wang, J. Zhu, Angew. Chem. Int. Ed. 2017, 56, 9577-9581; c) J. Hu, Z. Li, X. Zhang, Y. Han, Y. Liu, Y. Zhao, Y. Liu, P. Gong, Org. Lett. 2018, 20, 2116-2119.

[9] a) H. Tsutsui, K. Narasaka, Chem. Lett. 1998, 28, 45-46; b) M. Kitamura, S. Zaman, K. Narasaka, Synlett 2001, 974-976; c) H. Tsutsui, M. Kitamura, K. Narasaka, Bull. Chem. Soc. Jpn. 2002, 75, 1451-1460; d) S. Zaman, M. Kitamura, K. Narasaka, Bull. Chem. Soc. Jpn. 2003, 76, 1055-1062; e) M. Kitamura, K. Narasaka, Chem. Rec. 2002, 2, 268-277. 
[10] a) A. M. Berman, J. S. Johnson, Synlett 2005, 11, 1799-1801; b) T. J. Barker, E. R. Jarvo, J. Am. Chem. Soc. 2009, 131, 15598-15599; c) Y. H.Chen, S. GraßI, P. Knochel, Angew. Chem. Int. Ed. 2017, 56, 11081111; d) H.-B. Yang, S. R. Pathipati, N. Selander, ACS Catal. 2017, 7 , 8441-8445; e) J. Xiao, Y. He, F. Ye, S. Zhu, Chem 2018, 4, 1-13.

[11] J. Derosa, V. T. Tran, V. A. van der Puyl, K. M. Engle, Aldrichimica Acta 2018, 51, 21-32.

[12] a) J. Derosa, V. T. Tran, M. N. Boulous, J. S. Chen, K. M. Engle, J. Am. Chem. Soc. 2017, 139, 10657-10660; b) J. Derosa, V. A. van der Puyl, V. T. Tran, M. Liu, K. M. Engle, Chem. Sci. 2018, 9, 5278-5283.
[13] H. Lv, L.-J. Xiao, D. Zhao, Q.-L. Zhou, Chem. Sci. 2018, 9, 6839-6843.

[14] T. Deguchi, H.-L. Xin, H. Morimoto, T. Ohshima, ACS Catal. 2017, 7, 3157-3161.

[15] M. Newcomb, Radical Kinetics and Clocks. In Encyclopedia of Radicals in Chemistry, Biology and Materials, C. Chatgilialoglu, A. Studer, Eds. John Wiley \& Sons, Ltd.: 2012 